\title{
Critical fluctuations and complex spinodal points
}

\author{
Xin $\mathbf{A n}{ }^{* \dagger}$ \\ Department of Physics, University of Illinois, Chicago, IL 60607, USA \\ E-mail: xan2@uic.edu \\ David Mesterházy \\ Albert Einstein Center for Fundamental Physics, Institute for Theoretical Physics, University of \\ Bern, 3012 Bern, Switzerland \\ E-mail: mesterheitp.unibe.ch
}

\section{Mikhail A. Stephanov}

Department of Physics, University of Illinois, Chicago, IL 60607, USA

E-mail: misha@uic.edu

The experimental signatures of the QCD critical point rely on the universal singular behavior of the equation of state at the critical point. Therefore, we study singularities of the universal scaling equation of state of the $\phi^{4}$ theory, or the Ising model. We focus on the relation between spinodal points that limit the domain of metastability for temperatures below the critical temperature, i.e., $T<T_{\mathrm{c}}$, and Lee-Yang edge singularities that limit the domain of analyticity around the point of zero magnetic field $H$ for $T>T_{\mathrm{c}}$. The extended analyticity conjecture (due to Fonseca and Zamolodchikov) that for $T<T_{\mathrm{c}}$ the Lee-Yang edge singularities are the closest singularities to the real $H$ axis have interesting implications, in particular, that the spinodal singularities do not lie on the real $H$ axis. We find that the Ising model parametric equation of state obtained in the $\varepsilon=4-d$ expansion, as well as the related $O(N)$ model equation of state at large $N$, are both nontrivially consistent with this conjecture, and analyze the reason for the difficulty of addressing this question using the $\varepsilon$-expansion. In particular, we resolve the long-standing paradox associated with the fact that the vicinity of the Lee-Yang edge singularity is described by Fisher's $\phi^{3}$ theory, which is nonperturbative even for $d \geq 4$ where the equation of state of the $\phi^{4}$ theory is expected to become mean-field-like. We derive the Ginzburg criterion that determines the size of the region around the Lee-Yang edge singularity, where the mean-field theory no longer applies.

Critical Point and Onset of Deconfinement

7-11 August, 2017

The Wang Center, Stony Brook University, Stony Brook, NY

\footnotetext{
${ }^{*}$ Speaker.

${ }^{\dagger}$ This material is based on work supported by the U.S. Department of Energy, Office of Science, Office of Nuclear Physics under Award Number DE-FG0201ER41195 and is funded by the Swiss National Science Foundation.
} 


\section{Introduction}

One of the most intriguing questions about the QCD phase diagram - the existence and location of the QCD critical point - remains open. The search for the QCD critical point is a major component of the Beam Energy Scan (BES) program at the Relativistic Heavy Ion Collider (RHIC) at Brookhaven National Laboratory and involves theoretical as well as experimental effort. Due to the universality of critical phenomena, the knowledge of the scaling equation of state of the critical $\phi^{4}$ field theory (in the Ising universality class), could be applied to the study of the QCD critical point $[1,2]$. The scaling equation of state, which can be characterized by a universal function of a single argument - a scale-invariant combination of two relevant variables, is still not known exactly. Nevertheless, a lot is known about the equation of state [3] based on analytical and numerical studies. In particular, the equation of state near the upper critical dimension, $d=4$, is understood via the perturbative Wilson-Fisher fixed point using the $\varepsilon=4-d$ expansion [4-6].

In this contribution, which is a summary of Ref. [7], we focus on the analytic properties of the universal equation of state in the scaling regime near the Ising $/ \phi^{4}$ critical point as a function of a complex magnetic field $H$. Our main starting points are the pioneering work by Lee and Yang [8] connecting complex singularities of the thermodynamic functions to the phase transitions and the subsequent observation by Fisher that the singularity at the complex Lee-Yang edge point corresponds to the critical point in the $\phi^{3}$ theory [9] even though the $\phi^{4}$ theory determines the position of the Lee-Yang edge point. The apparent contradiction between the conclusions of Fisher's analysis and the $\varepsilon$ expansion around $d=4$ will be addressed.

Analyticity of the equation of state allows one to connect high- and low-temperature domains near the critical point. In particular, using the mean-field equation of state one can show that the Lee-Yang edge singularities, which reside on the imaginary magnetic field axis, are analytically connected to singularities that limit the domain of metastability - so-called spinodal singularities, which reside on another Riemann sheet reachable by analytic continuation through the branch cut along the real magnetic field axis. The position of these singularities on the real axis, however, is an artifact of the mean-field approximation. In fact, in $4-\varepsilon$ dimensions the position of the spinodal point shifts into the complex plane by an amount of order $\varepsilon^{2}$. We analyze this phenomenon in the framework of the $\varepsilon$ expansion employing parametric representations of the equation of state $[10,11]$, and confront the extended analyticity conjecture advanced by Fonseca and Zamolodchikov [12], which states that the complexified spinodal point is the nearest singularity to the real axis of the magnetic field. Our work could help develop better parametrizations of the equation of state by taking into account its correct analytic properties, especially in the metastable region on the first-order side of the critical point, which is important for the experimental search for the QCD critical point. The knowledge of the complex singularities of the equation of state is also important for determining the position of the critical point using lattice Taylor expansion methods [13].

\section{Critical mean-field equation of state}

The scalar $\phi^{4}$ theory in $d$ dimensions can be defined by the Euclidean action

$$
\mathscr{S}=\int d^{d} x\left[\frac{1}{2}\left(\partial_{\mu} \phi\right)^{2}+\frac{r_{0}}{2} \phi^{2}+\frac{u_{0}}{4 !} \phi^{4}-h_{0} \phi\right]=\frac{6}{u_{0}} \int d^{d} x\left[\frac{1}{2}\left(\partial_{\mu} \Phi\right)^{2}+V(\Phi)\right]
$$


with the rescaled potential

$$
V(\Phi)=\frac{t}{2} \Phi^{2}+\frac{1}{4} \Phi^{4}-H \Phi,
$$

where $\Phi=\sqrt{u_{0} / 6} \phi, H=\sqrt{u_{0} / 6} h_{0}$ and $t=r_{0} \sim T-T_{\mathrm{c}}$. In the mean-field (saddle-point) approximation justifiable for $d \geq 4$, the coupling $u_{0}$ runs into the Gaussian IR fixed point and becomes arbitrary small as $\xi \rightarrow \infty$, thus the expectation value of the field $\langle\Phi\rangle=M$ minimizing the potential (2.2) simply satisfies

$$
V^{\prime}(M)=-H+t M+M^{3}=0 .
$$

The implicit multivalued function $M(t, H)$ defined by Eq. (2.3) represents the mean-field equation of state of the $\phi^{4}$ theory. In the mean-field case, the correlation length is given by $\xi^{-2}=V^{\prime \prime}(M)=$ $t+3 M^{2}$, which is finite for all real values of $H$ when $t>0$. However, solving for $V^{\prime}(M)=V^{\prime \prime}(M)=$ 0 , we find points on the imaginary axis, where $\xi \rightarrow \infty$ for $t>0$ :

$$
M_{\mathrm{LY}}= \pm \frac{1}{\sqrt{3}} i t^{1 / 2} \quad \text { and } \quad H_{\mathrm{LY}}= \pm \frac{2}{3 \sqrt{3}} i t^{3 / 2} .
$$

These branch points of $M(H)$, known as Lee-Yang (LY) edge singularities, terminate cuts that lie on the imaginary $H$ axis (according to the Lee-Yang theorem $[8,14]$ ). They pinch the real $H$ axis as $t \rightarrow 0$. On the other hand, below the critical temperature, $t<0$, the mean-field approximation predicts that the correlation length diverges at real values of $M$ and $H$. These so-called spinodal points are located on the metastable branch and limit the domain of metastability [15, 16].

Since the critical equation of state is scaling [15], one may introduce properly normalized scaling variables $w=H t^{-3 / 2}$ and $z=M t^{-1 / 2}$, and rewrite the mean-field equation of state Eq. (2.3) as

$$
w=F(z)=z\left(1+z^{2}\right),
$$

The inverse of the (mean-field) function $F(z)$, i.e., $z(w)$, is multivalued and has three Riemann sheets associated with the high- and low-temperature regimes of the mean-field equation of state. The principal sheet, which represents the equation of state $M(H)$ for $t>0$, features two branch points located on the imaginary axis in the complex $w$ plane, corresponding to the Lee-Yang edge singularities at imaginary values of $H$, i.e.,

$$
w_{\mathrm{LY}}= \pm \frac{2 i}{3 \sqrt{3}} .
$$

Going under either one of the associated branch cuts, e.g., by following the path shown in Fig. 1, one arrives on the secondary sheet, which corresponds to the metastable branch of the equation of state at $t<0$. The same branch point in Eq. (2.6) viewed from this sheet represents the spinodal point located at real negative $H$, i.e.,

$$
H_{\mathrm{sp}}=\mp\left|w_{\mathrm{LY}} t^{3 / 2}\right|, \quad t<0 .
$$

To arrive on the stable $t<0$ branch where $H>0$, one has to follow the circular path further in the anticlockwise direction, as shown in Fig. 1 (right). We conclude that, in the mean-field approximation, the spinodal points and the Lee-Yang edge singularities are manifestations of the same singularities of the scaling equation of state $z(w)$. 


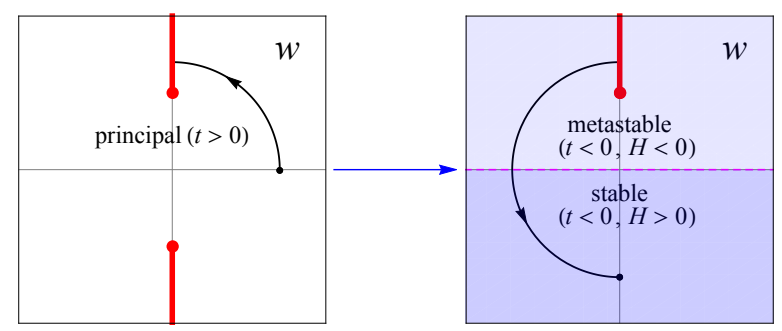

Figure 1: Analytic continuation $t \rightarrow-t$ from the principal, i.e., high-temperature sheet (left panel) to the low-temperature sheet (right panel) of the mean-field scaling function $z(w)$ in Eq. (2.5) with $w=H t^{-3 / 2}$. Starting from $H>0$ and $t>0$, keeping $H>0$ and $|t|$ fixed we rotate the phase $\arg t$ from 0 to $-\pi$ and trace the corresponding movement of the variable $w$ along the shown circular path. The principal sheet features a pair of Lee-Yang branch cuts along the imaginary $w$ axis, which terminate in the Lee-Yang edge singularities. Going through the cut we enter the metastable low-temperature branch $(H<0, t<0)$. One reaches the stable branch $(H>0, t<0)$ when $\arg t=-\pi$. From there one can also reach metastable branch $H<0$ by rotating $\arg H$ from 0 to $\pm \pi$, which changes $\arg w$ by $\pm \pi$.

\section{Beyond the mean-field approximation}

For $d<4$, however, the coupling $u_{0}$ runs into the Wilson-Fisher (WF) IR fixed-point and becomes finite as $\xi \rightarrow \infty$. In particular for $\varepsilon=4-d \ll 1, u_{0}^{\mathrm{WF}}=\mathscr{O}(\varepsilon)$. Although the theory is nonperturbative when $d=3$, we can still address some general properties beyond the mean-field approximation.

Firstly, according to the Lee-Yang theorem $[8,14]$ the singularities (on the physical stable branches of the free energy) of the Ising model must be located on the imaginary axis of $H$. Thus compared to the mean-field theory, the Lee-Yang edge singularities $w_{\mathrm{LY}} \sim H_{\mathrm{LY}} t^{\beta \delta}$ (and their associated cuts) remain on the imaginary axis of the stable branch $t>0$. However, analyticity and scaling imply that the spinodal points are on the low-temperature branch $t<0$ at

$$
H_{\mathrm{sp}} \sim w_{\mathrm{LY}} t^{\beta \delta}=\mp\left|w_{\mathrm{LY}} t^{\beta \delta}\right| e^{ \pm i \pi(\beta \delta-3 / 2)}, \quad t<0,
$$

displaced from the (negative) real $H$ axis by a phase

$$
\Delta \phi=\pi\left(\beta \delta-\frac{3}{2}\right)
$$

which is nonzero for $d<4$ since $\beta \delta>3 / 2$.

Secondly, the conjugate points in Eq. (3.1) dictated by the Schwartz reflection principle must reside on different ancillary Riemann sheets, separated by a cut along the real $H$ axis. This is the Langer cut [17] terminating at a weak essential singularity of the Ising equation of state at $H=0$ for $t<0$, and is associated with the decay of the metastable vacuum. The rate of this decay gives the imaginary part of the magnetization (or free energy) for $H$ on the metastable branch at $t<0$, i.e., $\operatorname{Im} M(t, H) \sim \exp \left(-\right.$ const $\left./ \mathrm{u}_{0}|\mathrm{w}|^{3}\right)$ when $d=4$ and $w \ll 1$. The nonperturbative dependence on $u_{0}$ means that not only is this singularity absent in the mean-field equation of state, but it cannot 


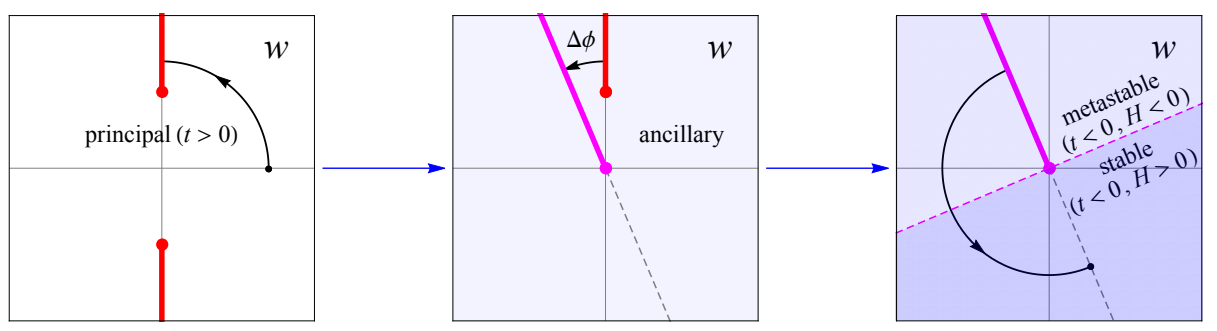

Figure 2: Analytic continuation $t \rightarrow-t$ from the principal, i.e., high-temperature sheet (left panel) to the low-temperature sheet (right panel) of the scaling function $z(w)$ of the Ising theory as conjectured by Fonseca and Zamolodchikov, where $w \sim H t^{-\beta \delta}$, while keeping the magnetic field $H>0$ fixed at $d=4-\varepsilon$. After analytic continuation the metastable branch $H<0$ can be accessed by rotating $H$ clockwise in the complex plane, while keeping $t<0$ fixed. The line representing the Langer cut is rotated away from imaginary axis by an angle $\Delta \phi$, cf. Eq. (3.2).

be seen at any finite order of the $\varepsilon$ expansion. As shown in Fig. 2, this cut can be reached from the stable low-temperature branch $(H>0, t<0)$ by rotating $H$ along a semicircle in the complex $H$ plane, such that $H \rightarrow-H$. The spinodal point can be found under the Langer cut. Thus it is natural to expect that the spinodal singularity (Lee-Yang edge singularity) is the closest singularity to the real axis (i.e., to the Langer cut). This is the essence of the "extended analyticity" conjecture put forward by Fonseca and Zamolodchikov [12].

Thirdly, one should note that the singular behavior near the Lee-Yang point is described by a $\phi^{3}$ theory [9] with upper critical dimension $d=6$. It has an IR fixed point occurring at imaginary values of the cubic coupling. Near the Lee-Yang point, $M-M_{\mathrm{LY}} \sim\left(H-H_{\mathrm{LY}}\right)^{\sigma}$ with $\sigma \approx 0.26$ in $d=4$ [18-21], which differs significantly from the mean-field value $\sigma=1 / 2$. The reason is that at any given value of $\varepsilon$ (and $t$ ), the magnitude of the fluctuations increases as we approach the Lee-Yang points and the theory becomes nonperturbative. We derive the Ginzburg criterion [7] to determine how close the Lee-Yang edge singularity can be approached before mean-field theory breaks down. The relative importance of fluctuations, is determined by the competition of the magnitude of the dimensionless cubic coupling $\widetilde{g}_{3} \equiv g_{3} \xi^{(6-d) / 2}$ and quartic coupling $\widetilde{u}_{0} \equiv$ $u_{0} t^{(d-4) / 2}=u_{0} t^{-\varepsilon / 2}$. Near $d=4$, for a generic value away from $w_{\mathrm{LY}}$ the condition is simply $\varepsilon \ll 1$. However, as $w \rightarrow w_{\mathrm{LY}}$ the correlation length diverges, fluctuations are enhanced, and the condition on $\varepsilon$ becomes more restrictive, i.e.,

$$
\widetilde{g}_{3} \sim \widetilde{u}_{0}^{1 / 2}\left|w-w_{\mathrm{LY}}\right|^{-(2+\varepsilon) / 8} \ll 1 .
$$

For $0<\varepsilon \ll 1$, this yields the following requirement

$$
\left|w-w_{\mathrm{LY}}\right| \gg \varepsilon^{2},
$$

where we replaced $u_{0}$ with its IR fixed-point value $u_{0}^{\mathrm{WF}} \sim \varepsilon$. Eq. (3.4) is the Ginzburg criterion that determines the size of the critical region around the Lee-Yang point. Inside this region the mean-field approximation breaks down and the correct scaling near that point is given by the fixed point of the $\phi^{3}$ theory, which is nonperturbative in $d=4$. 


\section{Singularities in the Ising theory and $\mathrm{O}(N)$-symmetric $\phi^{4}$ theory}

In this section we briefly discuss the major results of Ref. [7] on $d=4-\varepsilon$ Ising theory $(N=1)$ up to $\mathscr{O}\left(\varepsilon^{2}\right)^{1}$ and $\mathrm{O}(N)$-symmetric $\phi^{4}$ theory at large $N$ for various dimensions.

The perturbative calculation of the Ising equation of state has been done up to the third order in $\varepsilon$ [4-6]. For instance, in terms of the scaling variables $w \sim H t^{-\beta \delta}$ and $z \sim M t^{-\beta}$, the conventionally normalized equation of state can be written as

$$
w=F(z)=\sum_{n=0}^{\infty} F_{n}(z) \varepsilon^{n}
$$

where $F_{0}(z)$ is identical to the mean-field result Eq. (2.5). $F(z)$ is convenient to describe the analytic properties of the equation of state at finite $t>0$ and small $M$ (i.e., small $z$ ). However, the analyticity in the regime $M>0$ and small $t$ (i.e., large $z$ ) is obscured, since it corresponds to the behavior of $F(z) \sim z^{\delta}$ at large $z$ and the $\varepsilon$ expansion of $F(z)$ does not converge at large $z$. This problem is solved by the parametric representation $[10,11]$ of the equation of state where the analyticity is manifest in both regimes and the $\varepsilon$ expansion converges uniformly.

Using a variable transformation $(t, M) \rightarrow(R, \theta)$, the equation of state can be written as

$$
t=R k(\theta), \quad M=R^{\beta} m(\theta), \quad H=R^{\beta \delta} h(\theta),
$$

with analytic functions of the linear parametric model (LPM) [10] given by

$$
k(\theta)=1-\theta^{2}, \quad m(\theta)=\bar{m} \theta \quad \text { and } \quad h(\theta)=\bar{h}\left(\theta+h_{3} \theta^{3}\right),
$$

where $\bar{m}$ and $\bar{h}$ are normalization constants, $h_{3}=h_{30}+h_{31} \varepsilon+\mathscr{O}\left(\varepsilon^{2}\right) \cdot \bar{m}, \bar{h}$ and $h_{30}=-2 / 3$ are determined by matching the parametric model to the canonical equation of state [4-6], while $h_{31}$, on the other hand, cannot be determined by matching at order $\varepsilon^{2}$ or higher and it is common to set $h_{31}=0$ for simplicity $[5,6]$.

In terms of the linear parametric representation, the scaling variables $z$ and $w$ are given by

$$
z=\frac{\bar{z} \theta}{\left(1-\theta^{2}\right)^{\beta}} \quad \text { and } \quad w=\frac{\bar{w}\left(\theta+h_{3} \theta^{3}\right)}{\left(1-\theta^{2}\right)^{\beta \delta}}
$$

where $\bar{z}$ and $\bar{w}$ are normalization factors. We then arrive at the following expression for the inverse susceptibility

$$
F^{\prime}(\theta)=\frac{\bar{w}}{\bar{z}}\left(1-\theta^{2}\right)^{-\gamma} \frac{1+\left(2 \beta \delta+3 h_{3}-1\right) \theta^{2}+(2 \beta \delta-3) h_{3} \theta^{4}}{1-(1-2 \beta) \theta^{2}}
$$

It is convenient to consider its singularities as a function of $\theta^{2}$. Obviously there are two branchpoint singularities at $\theta^{2}=1$. For nonzero $\varepsilon$ in Eq. (4.5), the numerator has two zeros (labeled by $n=1,2$ ) while the denominator has one pole (labeled by $n=0$ ), all occurring at finite, albeit large, values of $\theta^{2}=\mathscr{O}\left(\varepsilon^{-1}\right)$. Indeed, these solutions can be expanded in powers of $\varepsilon$ where the

\footnotetext{
${ }^{1}$ We will briefly mention the results up to $\mathscr{O}\left(\varepsilon^{3}\right)$, which are discussed in detail in the Appendix of Ref. [7].
} 

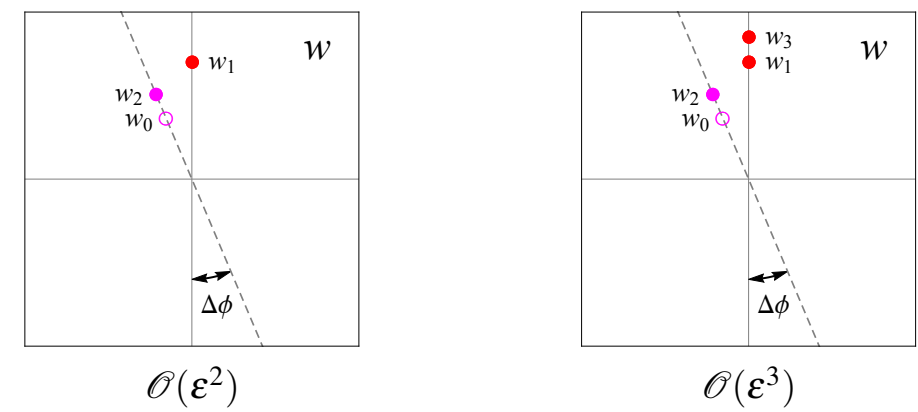

Figure 3: The position of the zeros $w_{n=1,2,3}$ (solid points) and the pole $w_{0}$ (open circle) of the parametrically represented inverse isothermal susceptibility $F^{\prime}(z)$ up to order $\mathscr{O}\left(\varepsilon^{2}\right)(l e f t)$ and $\mathscr{O}\left(\varepsilon^{3}\right)(r i g h t)$ in the complex $w$ plane. Note, only the singularities in the upper half of the complex $w$ plane are shown.

leading contribution appears at order $\varepsilon^{-1}$, i.e., $\theta_{n}^{2}=c_{n} \varepsilon^{-1}[1+\mathscr{O}(\varepsilon)]$. Expanding $w_{n} \equiv w\left(\theta_{n}\right)$ in $\varepsilon$ we arrive at

$$
w_{n}= \pm \frac{2 i\left(-\hat{c}_{n}\right)^{\frac{3}{2}-\beta \delta}}{3 \sqrt{3}}\left\{1+\mathscr{O}\left(\varepsilon^{2}\right)\right\}
$$

where $\hat{c}_{n} \equiv c_{n} /\left|c_{n}\right|$ and specifically,

$$
c_{0}=3, \quad c_{n}=3\left(\frac{9}{2} h_{31}+(-1)^{n} \sqrt{1+\left(\frac{9}{2} h_{31}\right)^{2}}\right), n=1,2 .
$$

Substituting Eq. (4.7) back to Eq. (4.6), we obtain

$$
\begin{aligned}
& w_{0}= \pm \frac{2 i}{3 \sqrt{3}}(-1)^{\frac{3}{2}-\beta \delta}\left\{1+\mathscr{O}\left(\varepsilon^{2}\right)\right\} \\
& w_{1}= \pm \frac{2 i}{3 \sqrt{3}}\left\{1+\mathscr{O}\left(\varepsilon^{2}\right)\right\}, \quad w_{2}= \pm \frac{2 i}{3 \sqrt{3}}(-1)^{\frac{3}{2}-\beta \delta}\left\{1+\mathscr{O}\left(\varepsilon^{2}\right)\right\} .
\end{aligned}
$$

The position of the singularities $w_{n=0,1,2}$ is shown schematically in Fig. 3 in the upper half of the complex $w$ plane. For comparison, we also show the $\mathscr{O}\left(\varepsilon^{3}\right)$ result in Fig. 3, where an additional zero $w_{3}$ emerges [7].

Note that the zero and the pole on the line of the Langer cut are within distance $\mathscr{O}\left(\varepsilon^{2}\right)$ from the Lee-Yang edge singularity, since $\beta \delta-3 / 2=\mathscr{O}\left(\varepsilon^{2}\right)$. Thus, according to the Ginzburg criterion in Eq. (3.4), these singularities and their position are nonperturbative, which is in agreement with the fact that we cannot fix the parameter $h_{31}$ determining their position within the $\varepsilon$ expansion. In addition, the higher order (i.e., $\varepsilon^{3}$ ) correction feeds down to the lower order terms of $w_{n}$, i.e., the extension of the $\varepsilon$ expansion to higher orders does not converge in the usual sense. It could be expected, in the spirit of Padé approximation, that those points will eventually coalesce into the Langer cut - a purely nonperturbative feature, which cannot be reproduced at any finite order of $\varepsilon$ expansion.

Although the results obtained in the $\varepsilon$ expansion are suggestive, the Ginzburg criterion (3.4) sets the limit on the precision that we can reach, $\varepsilon^{2}$, which is not sufficient to study the region 
between the Lee-Yang edge singularity and the Langer cut, for the purpose of rigorously testing the Fonseca-Zamolodchikov conjecture. Thus we also consider an alternative point of view on this question, by studying the generalization of the $\phi^{4}$ theory to the $N$-component theory with $\mathrm{O}(N)$ global symmetry, in particular, in the $N \rightarrow \infty$ limit referred as the spherical model.

Instead of the weak essential singularity in the $N=1$ case, the equation of state with $N>$ 1 has a power-law singularity in the low-temperature sheet which comes from the IR-divergent contributions of the Goldstone bosons. The Goldstone bosons become tachyonic and there is no metastable regime and tunneling involved in the decay of the false vacuum. Similar to the Langer cut in the Ising theory, the $N>1$ equation of state for $2<d<4$ has a "Goldstone cut" branching off from the origin and going along the negative real $H$ axis on the unstable branch. The lowtemperature image of the Lee-Yang edge singularities can be reached in the complex $H$ plane by going under the Goldstone cut onto an ancillary Riemann sheet, away from the cut by the angle $\Delta \phi=(d+2) / 2(d-2)$ for $2<d<4$. In fact, the situation is similar to the scenario shown in Fig. 2. These singularities become the spinodal points at $d=4$ when the equation of state at $N \rightarrow \infty$ takes the mean-field form (2.5).

Since there are no singularities in the equation of state of the spherical model $(N=\infty)$ and since $\Delta \phi \sim \mathscr{O}(\varepsilon)$, the Fonseca-Zamolodchikov scenario is realized in the $\mathrm{O}(N)$ model in the $N \rightarrow \infty$ limit. We have checked that (at least in $d=3$ ) this result is not affected by the leading $1 / N$ corrections [7]. Although the Fonseca-Zamolodchikov conjecture for the Ising critical equation of state is difficult to prove using the analytic methods considered, we can conclude that it is nontrivially consistent with the various systematic approximations to the equation of state beyond the mean-field level.

\section{Summary and discussion}

In this contribution we discussed the relationship between singularities of the universal scaling equation of state of the $\phi^{4}$ theory above and below the critical temperature [7]. The analyticity of equation of state as a function of $w$ would require the low-temperature manifestation of the LeeYang points to be points off the real axis by a phase $\Delta \phi=\pi(\beta \delta-3 / 2)$. Fonseca and Zamolodchikov put forward a conjecture that these are the closest singularities to the real $H$ axis, which is tested in the small- $\varepsilon$ and large- $N$ regimes. We derive the Ginzburg criterion to show that the $\varepsilon$ expansion must break down and the equation of state becomes nonperturbative in the region around the Lee-Yang point whose radius is proportional to $\varepsilon^{2}$ as $\varepsilon \rightarrow 0$. We have considered the parametric representation for Ising theory to order $\varepsilon^{2}$ and $\varepsilon^{3}$, and have shown that the singularities we find are consistent with the Fonseca-Zamolodchikov conjecture and the Ginzburg criterion. We point out that the equation of state of the $\mathrm{O}(N)$-symmetric $\phi^{4}$ theory satisfies the Fonseca-Zamolodchikov conjecture in the large- $N$ limit.

The absence of the spinodal singularities at real $H$ can be related to metastability: due to the critical slowing down near the thermodynamic singularity, it is impossible to realize the equilibration to such a critical state as the lifetime of the metastable state is finite. Indeed, the decay rate of the metastable state, which is controlled by the (small) coupling $u_{0}$ is no longer exponentially suppressed at the spinodal point. More precisely, the rate near the spinodal point for small $\widetilde{u}_{0}$ is given asymptotically by $\exp \left[-\operatorname{const}\left(w-w_{\mathrm{LY}}\right)^{(6-d) / 4} / \widetilde{u}_{0}\right][22,23]$, i.e., exponential suppression disappears in the Ginzburg region. This is to be expected since the fluctuations leading to the decay 
become important in that region. The fact that the shift of the spinodal singularity into the complex $H$-plane is also due to fluctuation contribution to the "gap" exponent $\beta \delta$ also suggests that the shift is related to metastability. It would be interesting to establish a more quantitative relation between this phenomenon and the Fonseca-Zamolodchikov conjecture.

This study may have implications for the understanding of the QCD phase diagram and the search for the QCD critical point in heavy-ion collisions. In particular, it may help better understand the evolution of the QCD fireball past the first-order phase transition in the vicinity of the critical point where the spinodal singularities may play a role [24,25].

\section{References}

[1] M. A. Stephanov, K. Rajagopal, and E. V. Shuryak, "Signatures of the tricritical point in QCD," Phys. Rev. Lett. 81 (1998) 4816, arXiv: hep-ph/ 9806219.

[2] M. A. Stephanov, "QCD phase diagram and the critical point," Prog. Theor. Phys. Suppl. 153 (2004) 139, arXiv: hep-ph $/ 0402115$. [Int. J. Mod. Phys. A 20 (2005) 4387].

[3] A. Pelissetto and E. Vicari, "Critical phenomena and renormalization group theory," Phys. Rep. 368 (2002) 549, arXiv: cond-mat/ 0012164.

[4] E. Brézin, D. J. Wallace, and K. G. Wilson, "Feynman graph expansion for the equation of state near the critical point (Ising-like case)," Phys. Rev. Lett. 29 (1972) 591.

[5] D. J. Wallace and R. K. P. Zia, "The $\varepsilon$-expansion and parametric models for the Ising equation of state in the critical region," Phys. Lett. A 46 (1973) 261.

[6] D. J. Wallace and R. K. P. Zia, "Parametric models and the Ising equation of state at order $\varepsilon^{3}$," $J$. Phys. C 7 (1974) 3480.

[7] X. An, D. Mesterházy, and M. A. Stephanov, "On spinodal points and Lee-Yang edge singularities," ArXiv e-prints (2017), arXiv:1707.06447 [hep-th] .

[8] T. D. Lee and C.-N. Yang, "Statistical theory of equations of state and phase transitions. II. Lattice gas and Ising model," Phys. Rev. 87 (1952) 410.

[9] M. E. Fisher, "Yang-Lee edge singularity and $\phi^{3}$ field theory," Phys. Rev. Lett. 40 (1978) 1610.

[10] P. Schofield, "Parametric representation of the equation of state near a critical point," Phys. Rev. Lett. 22 (1969) 606.

[11] B. D. Josephson, "Equation of state near the critical point," J. Phys. C 2 (1969) 1113.

[12] P. Fonseca and A. Zamolodchikov, "Ising field theory in a magnetic field: Analytic properties of the free energy," J. Stat. Phys. 110 (2003) 527, arXiv: hep-th/ 0112167.

[13] M. A. Stephanov, "QCD critical point and complex chemical potential singularities," Phys. Rev. D 73 (2006) 094508, arXiv: hep-lat/0603014.

[14] C.-N. Yang and T. D. Lee, "Statistical theory of equations of state and phase transitions. I. Theory of condensation,” Phys. Rev. 87 (1952) 404.

[15] M. E. Fisher, "The theory of condensation and the critical point," Physics 3 (1967) 255.

[16] K. Binder, "Theory of first-order phase transitions," Rep. Prog. Phys. 50 (1987) 783.

[17] J. S. Langer, "Theory of the condensation point," Annals Phys. 41 (1967) 108. [Annals Phys. 281 (2000) 941]. 
[18] F. Gliozzi and A. Rago, "Critical exponents of the 3d Ising and related models from conformal bootstrap," JHEP 10 (2014) 042, arXiv:1403.6003 [hep-th] .

[19] J. A. Gracey, "Four loop renormalization of $\phi^{3}$ theory in six dimensions," Phys. Rev. D 92 (2015) 025012, arXiv:1506.03357 [hep-th].

[20] X. An, D. Mesterházy, and M. A. Stephanov, "Functional renormalization group approach to the Yang-Lee edge singularity," JHEP 07 (2016) 041, arXiv:1605.06039 [hep-th] .

[21] L. Zambelli and O. Zanusso, "Lee-Yang model from the functional renormalization group," Phys. Rev. D 95 (2017) 085001, arXiv:1612.08739 [hep-th] .

[22] A. Z. Patashinskii and B. I. Shumilo, "Metastable systems near the instability region," Sov. Phys. Solid State 22 (1980) 655.

[23] C. Unger and W. Klein, "Nucleation theory near the classical spinodal," Phys. Rev. B 29 (Mar, 1984) 2698-2708. https://link.aps.org/doi/10.1103/PhysRevB.29.2698.

[24] J. Randrup, "Spinodal decomposition during the hadronization stage at RHIC?," Phys. Rev. Lett. 92 (2004) 122301, arXiv: hep-ph/0308271.

[25] C. Sasaki, B. Friman, and K. Redlich, "Density fluctuations in the presence of spinodal instabilities," Phys. Rev. Lett. 99 (2007) 232301, arXiv: hep-ph/ 0702254. 